\title{
Attention to Effects of Different Cross-Cultural Levels in User Research Method's Interface: Discipline or Nationality - Which Has Stronger Force?
}

\author{
Trang Thu Tran ${ }^{1}$ and Kun-Pyo Lee ${ }^{2}$ \\ Department of Industrial Design, KAIST, 335 Gwahangno, Yuseong-gu, Daejeon, \\ 305-701, South Korea \\ its2trang@gmail.com, kplee@kaist.ac.kr
}

\begin{abstract}
In the recent years, design research have developed a long way in investigating about the users and their contexts. It was aimed to challenge the way 'cross-culture influence' has been considered in design research field: should there be better way than profiling users solely based on nationality in multinational research project for product specification. Major findings through this research included (1) Nationality factor influenced remarkably on user performance but not much on user attitude. In contrast, discipline factor influenced significantly on every elements of user participation. (2) The gaps of user attitude and performance with nationality as the function maintained same levels while the effects from discipline factor intensified upon the increase of task complexity and change of ask characteristic. Overall, Discipline factor dominated Nationality factor, insisting on the importance of considering different levels of participants' cultures in designing the interface of user experience research methods.
\end{abstract}

Keywords: design research methodology, generative session, cross culture.

\section{Introduction}

Generative Session technique is a form of participatory design. It was first introduced by Elizabeth B-N Sanders in 1992 and was later described by the author as "these techniques can reveal tacit knowledge and expose latent needs". This technique is very different to the prior invented methods such as usability tests (in which users "do" something) and questionnaire or interview (in which users "say" something). In Generative sessions, users are the creators, who "make" designerly artefacts to express aspects of their situation, life, worries, and joys, etc. ${ }^{1}$

The method was inspired by the designers' ideation and communication activities. Furthermore it was based on the belief that "all people can project and express their needs, wants and aspirations through the use and interpretation of ambiguous visual stimuli.". These two points were considered as the key points and core innovativeness of the method.

${ }^{1}$ The concept of users "say-do-make" is mentioned by Sanders (1999) in her model: UX method and level of user experience knowledge. 


\section{Cultural Effects on User Research and Cross-Culture}

\subsection{Cultural Effects on User Research}

Based on the study on "culture and layers of influence on design research", Tran (2008) suggested the model of stimuli to cognitive programming as following.

There have been much attention paid to the cross-cultural effects in interface design and UX research in the recent years, in which researchers have been focusing on the effects of the middle layer: socio-cultural stimuli. Though socio-culture can be defined in multiple levels of groups (eg. Nationality, local area, career discipline, hobby, age, social class, etc.), most of the studies only have focused on the national culture level. There has been little study on the effects of sub-culture level. This initiated my question: further than national culture, can there be other level of socioculture that makes stronger effects on user participation?

\subsection{Cross-Socio Cultural Factors on User Research}

According to psychologists, behaviours and cognition can be differentiated by occupations - a mind formation and changing process. Such process is done and amplified through education and daily practice (Gardner, 2006). As mention in previous section, the key "interface" of Generative session technique include holistic thinking,

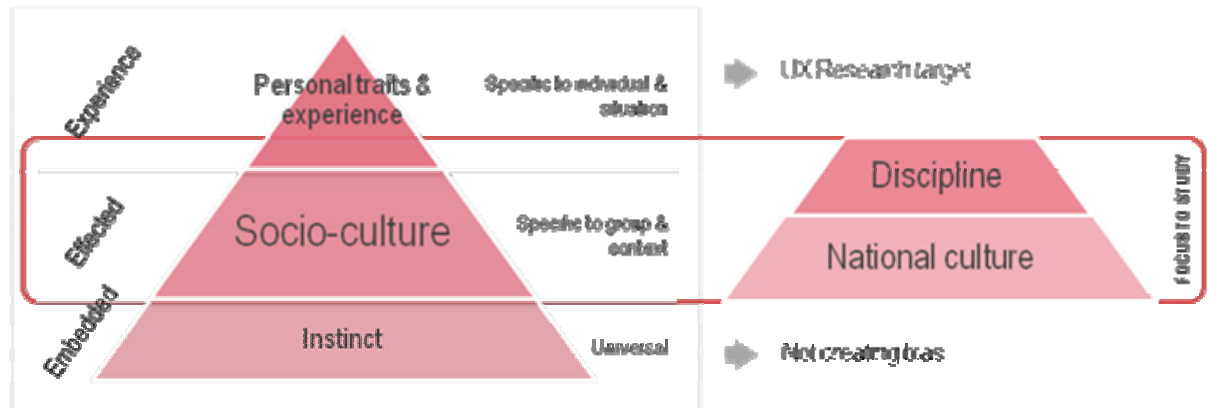

Fig. 1.

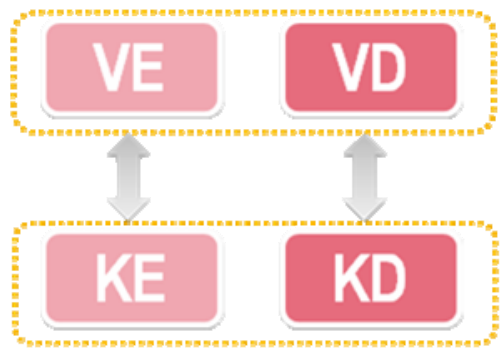

National gap dominates?
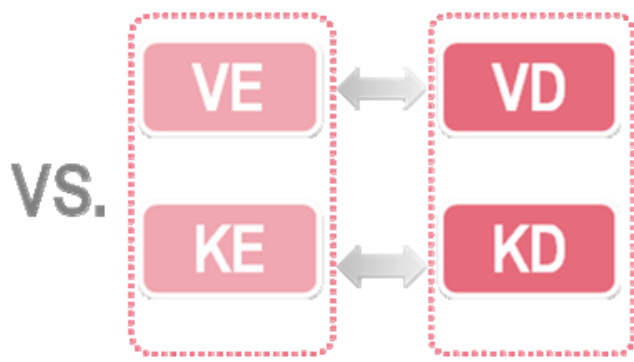

Disciplinary gap dominates?

Fig. 2. 
ambiguous terms, and richly visual interaction, question can be raised for this method: how this method is affected by the responses of participants from different groups of disciplines, which have different levels of intensiveness in daily practice with ambiguity and rich visual materials.

The experiment was carried out to answer the question: Among the two (major) factors (NAT and DIS), which one does generate more significant differences?

\section{Methods}

\subsection{Participants}

A proportionate, stratified, random sample was employed. Totally there were 60 participants recruited and divided into four groups of 15 .

All the Korean participants were students in various departments of Korea Advanced Institute of Science and Technology, South Korea. The Vietnamese-Designer participants were recruited from one design consultant company and the University of Architecture and Design of Ho Chi Minh City, Vietnam. The Vietnamese-Engineer participants were randomly recruited from the Institute of Environmental Technology located in Ho Chi Minh City, Vietnam.

The basic criteria for all participants were within the age range 20-30 and have at least 3 years experience in the career discipline. The latter condition was to ensure all participants had at least completed the basic skill training period in each discipline, so that the effects of discipline factor is significant to observe. There was a fairly equal distribution of genders in each group ( 7 or 8 among 15 participants belonged to one gender).

\subsection{Experiments}

The participants were asked to perform two tasks in Generative session designed following the experiment done by Sanders and Stappers in $2003^{2}$. In the first task, the participants were provided 120 trigger images and 90 words and were requested to select and arrange the images and words (among the provided triggers) to create a

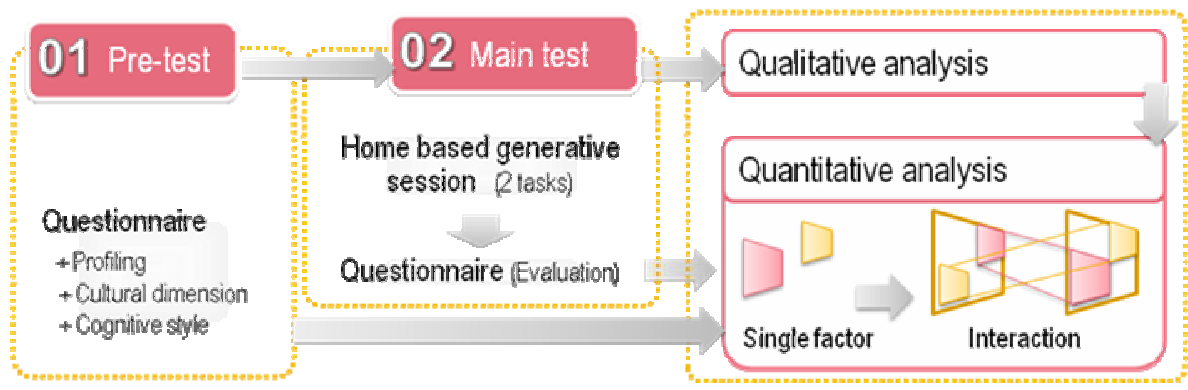

Fig. 3. Experiment scheme

\footnotetext{
${ }^{2}$ The experiment was conducted by the method inventors to test several combinations of elements in Generative sessions and to give the guideline to tune the method.
} 
collage that representing their experience in taking photos. In the second task, they were requested to choose an image that best represents their ideal experience of taking photos as the starting point. From the starting point, they were to expand it into a brainstorming map. Both tasks were expected to be done in a poster format.

The double task application was to check the effects in different levels of task complexity. Task 1 was claimed as the basic level among generative session techniques. Task 2 was stated as more difficult because the participants were not aided with triggers and they had to generate the map/poster basically from nothing. In addition, the characteristic of task 2 is not as 'free-style' as task 1, i.e. requiring more analytical thinking.

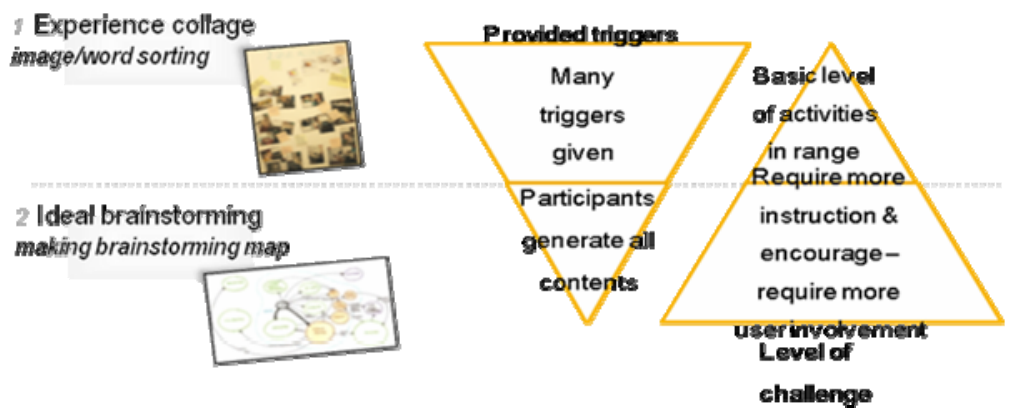

Fig. 4. Structure of task difficulties

Studies on user involvement in psychology and information system proved that there is a strong correlation between user attitude and user involvement, which is in turn correlated with the results. Moreover, it is possible to measure the involvement with questionnaires about user attitude and perception of how much the task relevant to their life experience. The latter notion however is not suitable in UX research as it is the main target of study ${ }^{3}$.

To predict the biased effects in user participation, we measured the user's attitude towards the task, based on the user evaluation, and their performance, based on the researcher's evaluation of participant generated results. User attitude includes three elements: time perception - does the user consider this task is waste of time or not, enjoyment perception - how much does the participant enjoy the task, and difficulty perception - how much difficult (s)he thinks the task is. The user performance was evaluated based on four elements: task completion time, imagery expressiveness level of expressiveness through imagery elements (from triggers and outsources), textual expressiveness - level of expressiveness through texts, and expression relevancy - how much relevant and specific user's expression of their experience is.

The participant generated posters were qualitatively analysis, using content analysis protocols. Their performance was translated into score in scale of 5 (1 for the lowest and 5 for the highest). The scales of score were the same in both attitude and performance. Finally, multiple fatorial ANOVA analyses were used to compare the user participation between two groups. 


\subsection{Analyzing Framework}

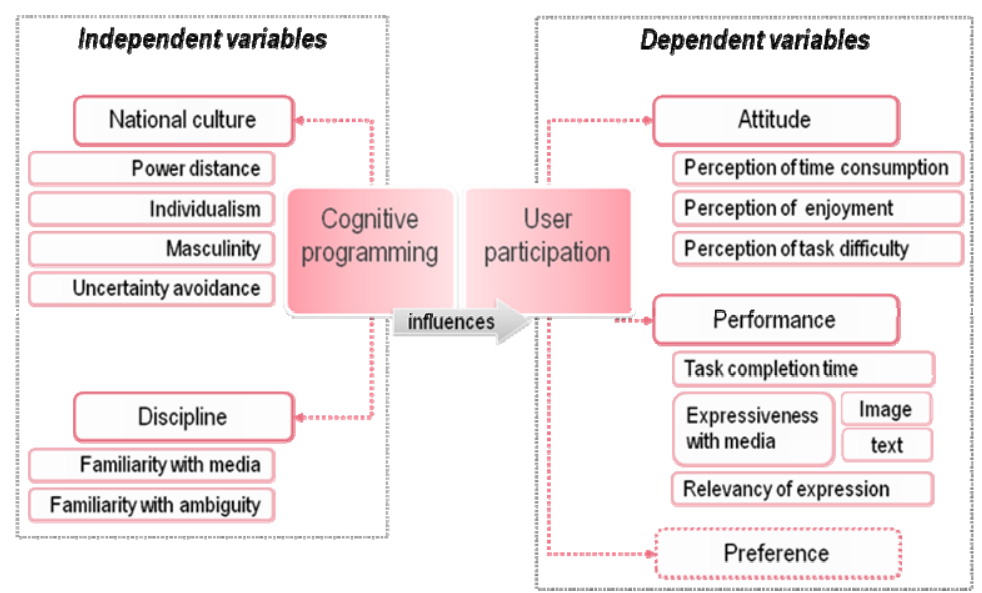

Fig. 5. Independent variables and dependent variables

\section{Results}

Major findings through this research included (1) Nationality factor influenced remarkably on user performance but not much on user attitude. In contrast, discipline factor influenced significantly on every elements of user participation. (2) The gaps of user attitude and performance with nationality as the function maintained same levels while the effects from discipline factor intensified upon the increase of task complexity and change of ask characteristic. Overall, Discipline factor dominated nationality factor, hypothesis [H0] was supported ${ }^{3}$, insisting on the importance of considering users' disciplines in designing 'Generative session' and ULX research.

A closer look into each element also revealed some complex influencing interactions: (1) the alternation of 'freshness' factor (research activity is different to daily practice thus interesting for participants) towards engineering groups' results but not on design groups, (2) the suspect of influence from nationality factor is not solely due to cultural dimension difference but rather socio-economic-education factors, and (3) among all user participation elements, 'perception of enjoyment' and 'task completion time' had odd pattern of influence which was caused by the interaction of NAT*DIS factors, this effects required more investigation.

Based on the findings and insights, guideline for method enhancement was suggested. Main recommendation focused on noting on what works with which group and suggesting different ways of presenting instruction and material provision for different groups of participants.

The guideline was divided in three parts: on cross national dimension, on cross disciplinary dimension, and for compound national-disciplinary profiling (case study of Korean-Vietnamese and Design-Engineering compounding matrix).

The following guidelines will be presented in table format. The left columns display the reference tags (of that particular guideline) that link back to findings from proceeding section.

\footnotetext{
${ }^{3}$ Details are provided in Table 2. Summary of hypotheses confirmation (section 5.5, page 69).
} 


\section{On cross national dimension}

Ref. tag Guideline and discussion

Both Korean and Vietnamese participants do not complain about their encountered difficulties with researchers so it is up to the researchers to check over the participants periodically and give aid when needed.

A1 Korean participants need to be encouraged by the beginning of the test. After that they can manage to complete the task on their own. Vietnamese participants prefer direct contact and need to be continuously encouraged and checked during their task conducting period. Therefore remote generative session is possible for Korean participants but will be inefficient to Vietnamese.

For Vietnamese users to be able to express themselves efficiently, more sensitizing should be provided to understand the topic and to get used to expressing

P1 techniques.

Pilot generative sessions with eventual increasing in complexity before real session are suggested.

Similar issue as previous point [P1]. Additional point is the national difference in performance also is influence by socio-national circumstances. Since they are

P2 inevitable, before designing research method, secondary research stage should include study of national education system and style, general media contents, etc. to gain understand of how people obtain and express opinions.

\section{On cross disciplinary dimension}

Ref. tag Guideline and discussion

There is a strong relation between enjoyment and perception of time consump-

tion. In order to gain user enjoyment, two approaches are available:

Carefully brief and psychologically prepare the participants, especially en-

A2, A3, gineers, about the process and that it may take long time. Showing examples of and A4 pre-done similar works with notion of time can be helpful.

Exploit the 'freshness' factor by integrate many beautiful visual elements in research package. However, pattern of how much visual elements and level of cuteness to be used should be studied further.

Consideration of participant's career discipline and expressing ability should be integrated in early stage of experimental design.

A5 With the same question, it is recommended to be delivered in different formats and manners to designers and engineers: designers will have more freestyle expressing task while engineers will have well-refined, structured task.

P3 The extra time spending by participants when they are involved deeply to the

(linked task should be predicted and informed to participants in advance so it will not

to A2) turn a pleasurable experience (of task conducting) to negative emotion (time concerning).

There should be consideration for profiling regarding users' skill and ability of expression when applying the generative session techniques. Consequently they should not be analyzed on the same base of assumption.

P4 Since UX researchers usually have design background, it probably will be hard for them to be completely objective while analyzing mixed designer and non-designer results. Therefore setting up separated criteria and assessment framework for each group of participants is suggested.

Although ambiguous stimuli are essential part of Generative session techniques, explicit level in task instruction and hints should be negotiated to a comprehensible level for engineers as ambiguous expession is unfamiliar to them. More study to determine the suitable level is advisable. 


\section{For compound national-disciplinary profiling (Korean-Vietnamese and Design- Engineering case)}

Korean design group appeared to have the best performance among the four groups overall. Their generated results were also the closest to researchers' normal expectation of collected data. Vietnamese engineering group had the lowest performance yet with very good attitude. Vietnamese designers did not perform as well as Korean designers but much better than the two engineering groups.

\begin{tabular}{|c|c|}
\hline Ref. tag & Guideline and discussion \\
\hline $\mathrm{E} 1$ & $\begin{array}{l}\text { Affluent visual element support to Korean designers is important to keep them } \\
\text { interest. (Note that the experiment in this study provided around } 140 \text { images, } \\
\text { which was already more than a standard package used in US or the Nether- } \\
\text { lands, yet it was not enough.) }\end{array}$ \\
\hline $\mathrm{E} 2$ & $\begin{array}{l}\text { If the task is conducted offline, bring in the convenience: provide pre-cut im- } \\
\text { ages and in sticker forms. However, there is a down side of cost and effort for } \\
\text { preparing such package, thus an online generative session will solve this two } \\
\text { matters. The trade off for online version is the technology barrier for some } \\
\text { participants. Furthermore, it is believe that people tend to be more creative with } \\
\text { hand-on tasks than computerized tasks. }\end{array}$ \\
\hline A6 & $\begin{array}{l}\text { Vietnamese designers had strong art based training so well structured expres- } \\
\text { sion (such as making process diagrams or maps) is not preferable to them. }\end{array}$ \\
\hline P6 & $\begin{array}{l}\text { Both engineering groups require much more sensitization than the method } \\
\text { already provides. Current method focuses on familiarize participants with the } \\
\text { topic. However, for non-designer users to be able to express themselves effi- } \\
\text { ciently, more sensitizing is needed for them to understand and be used to } \\
\text { expressing methods. Pilot generative sessions with eventual increasing in com- } \\
\text { plexity before real session are suggested. In addition, researchers can include } \\
\text { animated or interactive step-by-step instruction of the expressing method and } \\
\text { topic to educate the participants how-to-express-your-experience. }\end{array}$ \\
\hline A7, P6 & $\begin{array}{l}\text { Except for Korean designers, all other groups had problem (in different levels) } \\
\text { with interpreting the request in task } 2 \text {. For countries with high uncertainty } \\
\text { avoidance, an abstractive request such as 'talk about your ideal experience of } \\
\text { xyz' as in original method is not suitable and bring in unpleasant. A consider- } \\
\text { able way to rephrase the question is to provide them more information of the } \\
\text { circumstance. The procedure is like: } \\
\text { (1) provide the participants the descriptions of a certain ideal circumstance } \\
\text { or an ideal scenario, then } \\
\text { (2) ask for their response upon that scenario: "Have you ever experience this } \\
\text { situation/experience? If yes, tell us more detail of that time. If no, what would } \\
\text { you consider and do if you were in that case? Imagine your case and tell us." }\end{array}$ \\
\hline
\end{tabular}

\section{Application and Discussion}

The final accomplishment of this research was giving a guideline to enhance the method, showing practical experience and proven information to other researchers who intend to conduct related studies. It also raised some issues for further study of method enhancing.

Through this research, the upper level of mental programming stimuli (sub-culture discipline) was found to override the lower level (national culture). This result, firstly, 
was against the conventional belief in anthropology field that lower level always dominates upper level of the mental programming stimuli pyramid. It implied the change in human being's values and break of models within this ever-developing world. It further contributed to the dilemma in contemporary product development and business: globalization or localization, and to which level.

However, this study contained some limitations. Firstly, the results only apply for this specific case. More study needed for generalization of the theories as well as determine the pattern of influence. Secondly, the recruited participants were not equal in condition, such as the design participants were from different departments of design which may have different training programs and focus, as the same case as engineering participants. The impacts of compound variables are believed to exist.

Since this research only focused on one case study, with a two dimension matrix, it should be treated as pilot study and case study. The further work after this is suggested to be investigation of different combination of nations and disciplines, as well as different level of method complexity.

\section{References}

1. Sadler-Smith, E., Riding, R.J.: Cognitive style and instructional preferences. Instructional Science 27, 355-371 (1997)

2. Sanders, E.B.-N.: Postdesign and Participatory Culture. In: Proceeding in Useful and Critical: The Position of Research in Design, University of Art and Design Helsinki (UIAH), Tuusula, Finland (1999)

3. Barki, H., Hartwick, J.: Rethinking the Concept of User Involvement. MIS Quarterly 13, 53-63 (1989)

4. Gardner, H.: Changing Minds: the Art and Science of Changing Our Own and Other People's Minds. Harvard Business School Press, Boston (2006)

5. Rijn, H., Bahk, Y., Stappers, P., Lee, K.P.: Three Factors for Contextmapping in East Asia: Trust, Control and Nunchi. CoDesign 2, 157-177 (2006)

6. Stappers, P.J., Sanders, E.B.-N.: Generative Tools for Context Mapping: Tuning the Tools. In: Proceeding in Third International Conference on Design \& Emotion, Taylor \& Francis, Loughborough (2003)

7. Stappers, P.J., Sanders, E.B.-N.: Tools for designers, Products for Users? The Role of creative Design Techniques in a Aqueezed-in Design Process (2003)

8. Jordan, P.W.: Designing Pleasurable Products - An Introduction to the new Human Factors. Taylor \& Francis Group, London (2002)

9. Schuler, Namioka: Participatory design. Lawrence Erlbaum, Mahwah (1993) 